\title{
PHOTOEMISSION OF Cd(0001) USING SYNCHROTRON RADIATION
}

\author{
E. SobCZAK*, P.O. Nilsson and K. KarLsSON \\ Chalmers University of Technology, Department of Physics, 41295 Göteborg, Sweden
}

\begin{abstract}
Photoemission spectra for the $\operatorname{Cd}(0001)$ surface in the $\bar{\Gamma}-\bar{M}$ azimuth were calculated using a multiple scattering method. The emission angle of the photoelectrons $\theta$ was kept constant and equal to $35^{\circ}$. The photon energy was varied from $20 \mathrm{eV}$ to $27 \mathrm{eV}$. The self-consistent potential used in the calculations was generated by the scalar-relativistic LMTO method. A modified image potential for the surface barrier potential was applied. The photoemission spectra show a strong surface state peak at an energy of about $1 \mathrm{eV}$ below the Fermi level.
\end{abstract}

PACS numbers: 79.60.Cn, 73.20.At, 71.25.Pi

Synchrotron radiation is very useful for $k$-resolved photoemission studies because of the tunability and the possibility of band mapping of the electron states (both surface and bulk electron states). Spectra can be measured by varying the emission angle of photoelectrons and/or by varying the photon energy. Experimental photoemission spectra for Cd using synchrotron radiation have not yet been published.

In this work we present photoemission spectra for the $\mathrm{Cd}(0001)$ surface in the $\bar{\Gamma}-\bar{M}$ azimuth calculated using a multiple scattering method [1, 2]. The emission angle of photoelectrons $\theta$ was constant and equal to $35^{\circ}$. The polar angle of the light was $45^{\circ}$. The photon energy varied from $20 \mathrm{eV}$ to $27 \mathrm{eV}$. The angle of light polarization was assumed to be $45^{\circ}$ relative to the plane of incidence. It means that both $\sigma$ and $\pi$ polarizations are incorporated in the spectra.

We used a self-consistent muffin-tin potential for Cd generated by the scalar-relativistic LMTO method [3]. The work function of $\mathrm{Cd}(0001)$ was assumed to be $4.0 \mathrm{eV}$. The energy-dependent imaginary part of the potential was in the range from -0.3 to $-0.1 \mathrm{eV}$ for the initial state and in the range -1.6 to $-1.0 \mathrm{eV}$ for the final state. It induced a broadening of the surface state by $0.3 \mathrm{eV}$.

A modified image potential was assumed for the surface barrier potential [4]. The distance between the first atomic layer and the image potential plane was chosen to be $1.486 \AA$, which means half of the Cd lattice constant. The real image potential was used up to $20 \AA$ outside the surface. Within a distance of $1.5 \AA$ from

*Permanent address: Institute of Physics, Polish Academy of Sciences, Al. Lotników 32/46, 02-668 Warszawa, Poland. 
the image potential plane, a third-order polynomial matching smoothly the inner and image real potentials was used. We assumed the imaginary part of the image potential to be zero, except in the range up to $3.5 \AA$ above the image potential plane. Here, a third-order polynomial function was used, matching smoothly the inner imaginary potential (equal to $-0.136 \mathrm{eV}$ ) to zero at the image potential.

The reflection and transmission coefficients for an electron scattered by a surface barrier potential were calculated. The program BARRIER [4] for electron energies in the ranges -272.0 to $-0.01 \mathrm{eV}$ and 0.01 to $30.0 \mathrm{eV}$ relative to the vacuum level was applied. Then, the surface barrier contribution to the photoelectron intensity was calculated in the multiple scattering program using new formulae proposed by Borstel and Thörner [5].

The calculated photoelectron spectra of $\mathrm{Cd}(0001)$ are shown in Fig. 1. The intensity was assumed to be zero above the Fermi level. The step in the spectra at the Fermi level was smeared by a Gauss function with FWHM $=0.4 \mathrm{eV}$ in order to simulate the instrumental broadening of the photoelectron spectra. A strong surface state peak is observed at an energy of about $1 \mathrm{eV}$ below the Fermi level. A similar surface state peak was previously observed experimentally for $\mathrm{Cd}(0001)$ using $\mathrm{HeI}(21.2 \mathrm{eV})$ and $\mathrm{NeI}(16.8 \mathrm{eV})$ radiation [6].

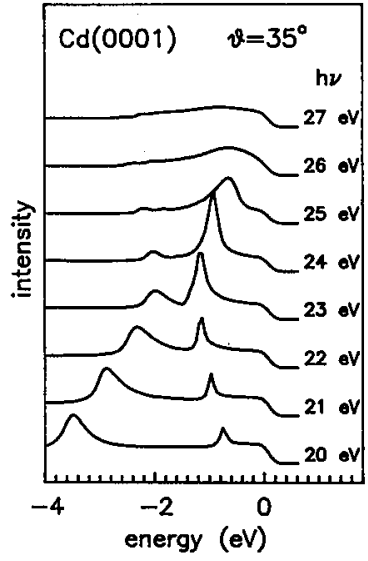

Fig.1

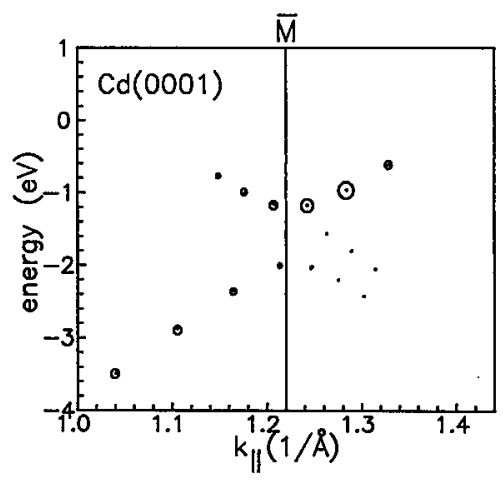

Fig. 2

Fig. 1. Pholoemission spectra for the $\mathrm{Cd}(0001)$ surface calculated for various photon energies.

Fig. 2. Dispersion of photoemission peaks versus parallel momentum $k_{\|}$. The diameters of the circles are proportional to the peak intensities.

The peak positions are plotted as a function of parallel momentum $k_{\|}$in Fig. 2. The intensity of the peaks changes rapidly with the parallel momentum $k_{\|}$and is indicated in Fig. 2 by the diameter of the circles. The energy position of peaks turns at the $\bar{M}$ point at $k_{\|}=1.22 \AA^{-1}$ and their intensities change asymmetrically around the $\overline{\mathbf{M}}$ point.

The surface state peak at about $-1.0 \mathrm{eV}$ is narrow and well separated from the 
bulk peak for the parallel momentum $k_{\|}$less than that at the $\bar{M}$ point $\left(1.22 \AA^{-1}\right)$. As soon as the parallel momentum $k_{\|}$passes the $\bar{M}$ point, the surface state peak increases by factor 2 and then rapidly smeares out. We interpret the increase in the intensity as caused by a resonance between surface and bulk states.

The reason for the very rapid changes in the momentum-resolved photoelectron spectra with small variations of the photon energy is the strong dispersion of the electron bands along the $\mathrm{\Gamma}-\mathrm{M}$ line [7]. Therefore, the $\mathrm{Cd}(0001)$ photoemission experiment, using synchrotron radiation, should be done using a photoelectron spectrometer with an acceptance angle as small as $\pm 0.5^{\circ}$ and applying small steps in the photon energy.

\section{Acknowledgements}

This work was supported by grants from the Swedish Natural Science Research Board.

\section{References}

[1] J.F.L. Hopkinson, J.B. Pendry, D.J. Titterington, Comput. Phys. Commun. 19, 69 (1980).

[2] C.G. Larsson, Ph.D. Thesis, CTH, Göteborg 1982.

[3] P.-O. Nilsson, E. Sobczak, K. Karlsson, T. Jarlborg, to be published.

[4] G. Malmström, J. Rundgren, Comput. Phys. Commun. 19, 263 (1980).

[5] G. Borstel, G. Thörner, Surf. Sci. Rep. 8, 1 (1988).

[6] H.S. Chauhan, L. Ilver, P.O. Nilsson, J. Kanski, U.O. Karlsson, Vacuum 41, 531 (1990).

[7] P. Blaha, K. Schwarz, P.H. Dederichs, Phys. Rev. B 38, 9368 (1988). 